Article

\title{
Study Protocol for a Randomized Double Blind, Treatment Control Trial Comparing the Efficacy of a Micronutrient Formula to a Single Vitamin Supplement in the Treatment of Premenstrual Syndrome
}

\author{
Hannah Retallick-Brown *, Julia Rucklidge and Neville Blampied \\ Department of Psychology, College of Science, University of Canterbury, Private Bag 4800, Christchurch 8041, \\ New Zealand; Julia.rucklidge@canterbury.ac.nz (J.R.); Neville.blampied@canterbury.ac.nz (N.B.) \\ * Correspondence: hahna.retallick-brown@pg.canterbury.ac.nz; Tel.: +03-364-2987 (ext. 7705); Fax: +03-364-2181
}

Academic Editor: James D. Adams

Received: 12 August 2016; Accepted: 15 November 2016; Published: 7 December 2016

\begin{abstract}
Background: The recent addition of Premenstrual Dysphoric Disorder (PMDD) to the Diagnostic and Statistical Manual (5th ed.) has highlighted the seriousness of this disorder. Many alternatives to psychoactive medication in the form of vitamins, minerals, and plant extracts have been trialled by women seeking a natural treatment approach. We plan to explore whether a well validated micronutrient formula, EMPowerplus Advanced, can outperform a recognized single nutrient treatment, vitamin B6, for the treatment of Premenstrual Syndrome (PMS). Methods: This will be a randomized treatment control study. Eighty women will be recruited and assigned to one of two treatment groups; EMPowerplus Advanced or vitamin B6. Baseline daily data will be collected for an initial two cycles, followed by three months of active treatment. A natural follow up will take place three cycles post treatment. Results: The primary outcome measure will be PMS change scores as based on results from the Daily Record of Severity of Problems (DRSP). The number of treatment responders for each of the two groups will yield a comparison score between the two treatments, with participants deemed as a responder if they show a total PMS score improvement of $50 \%$ from their baseline scores on the DRSP. Conclusion: If a micronutrient formula proves more effective for treating PMS, not only does it give women suffering from the condition a viable treatment option, but it may also suggest one cause of PMS; that is insufficient minerals and vitamins.
\end{abstract}

Keywords: premenstrual syndrome; premenstrual dysphoric disorder; treatment; vitamins; minerals

\section{Introduction}

The average woman will experience 13 menstrual cycles per year, corresponding to roughly 451 cycles over her lifetime and nearly 35 years of menstruation [1]. As many as 90\% [2] of women will experience mild physical or mental symptoms in the week leading up to menstruation, and for some the symptoms are severe. Approximately $20 \%$ to $40 \%$ of ovulating women will suffer from numerous symptoms, collectively termed; premenstrual syndrome (PMS) [3]. An even more symptomatic form of PMS, known as Premenstrual Dysphoric Disorder (PMDD) is found in $1.8 \%-5.8 \%$ of women [4]. For women meeting criteria for either PMS or PMDD the symptoms include: affective lability, irritability, anger, increased interpersonal conflict, depressed mood, anxiety, difficulty concentrating, fatigue, change in appetite, sleep disturbance, feelings of being out of control, less interest in normal activities as well as physical symptoms such as bloating, breast swelling, and joint pain [4]. The hormonal induced changes affect all women regardless of ethnicity or socio-economic status and place a burden on interpersonal relationships, mental health, and work productivity [3]. 
The American College of Obstetricians and Gynecologists (ACOG) recommend pharmacological treatment should be used as a first line intervention for PMDD [5]. Selective serotonin reuptake inhibitors (SSRIs) have demonstrated a small effect size in PMS change scores (standardized mean difference $=-0.36,95 \%$ CI -0.2 to -0.51 ) [6], although they are more effective in women with severe PMS/PMDD; showing a moderate effect size in overall symptom reduction in this population of women (standardized mean difference $=-0.53,95 \%$ CI 0.68 to -0.39 ) [7]. However, the use of anti-depressants in the treatment of PMS can, at times, cause significant side effects including nausea, loss of libido, fatigue, insomnia, dry mouth, gastrointestinal complaints, and tremors (among other problems) [8], which can cause many women to terminate treatment. Women are far more likely to terminate participation when in an active treatment group compared to women on placebo, with higher frequencies of side effects observed in the former group. However, results from SSRI trials have produced incomplete side effect results, with many studies neglecting to specify side effects [8]. The second line of pharmacological treatment, hormone replacement therapies, interrupt the process of sex hormone production, causing anovulation. Due to serious side effects including reduction in mineral bone density, induction of postmenopausal symptoms and induction of symptoms similar to those seen in PMS such as headaches, lowered mood, and muscle tenderness $[9,10]$, gonadotropin-releasing hormones $(\mathrm{GnRH})$ agonists are used sparingly. Meanwhile, oral contraceptives have shown poor treatment efficacy and are not a recommended treatment [11]. Women with less severe symptoms are advised to try lifestyle changes such as dietary alterations, exercising regularly, improving sleep length and quality, and alleviating stress [12]. As current frontline medical treatments for PMS can have significant side effects, there is a need for new forms of treatment.

Vitamins and minerals have a long history of being studied for the treatment of mental illness, usually one nutrient at a time [13]. A lack of specific vitamins and minerals (micronutrients) has been thought to be a causal factor in PMS and therefore their supplementation provides a possible treatment avenue [14]. Vitamin B6 (pyridoxine) has received much attention for its use in alleviating premenstrual symptoms. It has been hypothesized that B6 works, at least in part, through synthesizing serotonin, a neurotransmitter implicated in depression [15]. Numerous randomized controlled trials (RCTs) have studied the vitamin, finding promising results. For example, in a randomized crossover trial Doll, et al. [16] found pyridoxine to be effective in a dose as little as $50 \mathrm{mg}$ per day on the emotional symptoms of depression and irritability and on tiredness. Kashanian, et al. [17] in a placebo controlled trial showed that at $80 \mathrm{mg}$ per day pyridoxine exerted a significantly stronger effect on psychiatric symptoms compared to placebo, while both significantly reduced somatic symptoms. Wyatt, et al. [18] carried out a systematic review of the literature involving vitamin B6 and its use in the treatment of premenstrual syndrome. They noted that a number of studies were of poor quality but they did find evidence in support of the use of vitamin B6. In particular, the odds ratio for active treatment over placebo in alleviating overall premenstrual symptoms was 2.32 (95\% confidence interval 1.95 to 2.54 ).

While a number of other nutrients including calcium, vitamin E, magnesium have shown some evidence of efficacy in treating PMS [15,19], the overall results are generally modest, suggesting that the one disease one treatment model fails to take in the complexities of human nutrient requirements [20]. $1200 \mathrm{mg}$ of calcium a day over three menstrual cycles produced a $48 \%$ reduction in PMS symptoms compared to $30 \%$ in the placebo group [21]. Vitamin E has been indicated as a possible treatment, yet results have failed to reach significance [22]. Magnesium has shown variable results in treating the symptoms of PMS [23]. When combined with $50 \mathrm{mg}$ of vitamin B6, magnesium at a dose of $200 \mathrm{mg}$ /day was effective on multiple PMS symptoms despite a relatively short treatment duration of one month [24].

Given that micronutrients are implicated in a range of essential brain functions from neurotransmitter synthesis to powering brain metabolism [25] and work synergistically, there is a growing body of literature exploring a much greater combined approach. Indeed, broad spectrum micronutrient formulas have been found to be effective in treating a multitude of symptoms typically seen in PMS such as irritability, low mood, poor sleep and anger [26]. There is a 
growing body of literature demonstrating the benefit of using broad spectrum micronutrients in the treatment of a variety of psychological/psychiatric conditions such as anxiety, depression, bipolar, attention-deficit/hyperactivity disorder, and obsessive compulsive disorder in both adults and children and this approach is proving to be far more successful than any single nutrient alone [26]. Previous research has already explored the use of a multi-ingredient formula in the treatment of PMS [27-29]. However, the formula, "Optivite", contained high doses of both magnesium (250 mg/day) and vitamin B6 (300 mg/day) when taken at the full recommended dosage. Single nutrient doses as high as those seen in Optivite can be considered a standalone treatment in their own right, and indeed have been used in other studies $[18,23,24]$. Therefore, the results have been somewhat obscured by the high single nutrient dosages; was it the multi-ingredients or the vitamin B6/magnesium alone that resulted in improved PMS symptoms? The current study intends to clarify the efficacy of a broad spectrum formula in the treatment of PMS, using doses of single nutrients below the therapeutic levels of single nutrient treatments reported in previous studies.

The most studied broad spectrum micronutrient formula is called EMPowerplus (EMP+) which consist of 36 ingredients, the majority of which are minerals and vitamins (although it is sold variously as EMPowerplus Advanced, Daily Essential Nutrients and Q96). EMP+ has already shown efficacy in treating similar symptoms to those seen in the premenstrual phase such as low mood, anxiety, rage, insomnia and loss of control [26]. Given its efficacy in assisting with such symptoms, it is hypothesized that EMP+ will prove efficacious in women suffering from premenstrual complaints. This paper presents the study protocol for the Natural Treatment of Premenstrual syndrome (NTP) trial. This is a randomized treatment controlled trial that aims to investigate the effectiveness of micronutrients in the treatment of premenstrual syndrome (PMS). We hypothesize that women receiving EMP+ will experience greater improvements in PMS symptoms than women receiving vitamin B6.

\section{Methods}

\subsection{Study Design}

This study will follow women for a total of eight menstrual cycles; two cycles of pretreatment, three cycles of active treatment, and a three cycle natural follow up. It is a randomized, parallel group, double blind, treatment controlled trial of natural supplement interventions for moderate to severe premenstrual syndrome (PMS). We are currently enrolling 80 women from the greater Canterbury region (New Zealand). Recruitment and intervention are anticipated to occur over a 1.5-2 year time frame. Participants will be randomized to receive either a micronutrient formula, EMPowerplus Advanced (EMP+), or an active comparator treatment, vitamin B6.

\subsection{Study Aims}

We aim to investigate the efficacy of a micronutrient supplement (EMP+) in the treatment of PMS as measured by the Daily Record of Severity of Problems (DRSP) questionnaire. We are particularly interested in comparing EMP+ to an already efficacious single-ingredient natural treatment, vitamin B6. We hypothesize that those participants receiving EMP + will have greater improvement not only in their premenstrual symptoms but also on secondary measures of anxiety, depression, stress, sleep, sexual functioning, and quality of life. As well as looking at premenstrual symptoms change, we will record the number of treatment responders for each of the two treatments and compare the different rates of response. A participant will be deemed to be a responder if they show a total PMS improvement score of $50 \%$ or more as based on baseline scores from the DRSP. A further aim is to identify any mediation or moderation effects of stress sensitivity on PMS ratings.

\subsection{Participant Eligibility}

All participants must be over 18 years of age, experience regular menstrual cycles, must not be pregnant or breast feeding or attempting to become pregnant, and currently cannot be taking 
other medications for the treatment of PMS. If participants are using sex hormones other than the contraceptive pill they will also be excluded (these include gonadotropin-releasing hormone agonists, anti-estrogens, androgens, and estrogens specifically targeting PMS symptoms); however, as long as their contraceptive pill dosage stays the same throughout the study they are eligible to participate. Any dose or contraceptive pill change will be considered a protocol violation and the participant will therefore count as a drop out and will not be included in the per-protocol analyses. Final exclusion criteria include the presence of a current mood disorder (other than PMDD), a neurological disorder involving brain or other central nervous system function (e.g., epilepsy), evidence of untreated or unstable thyroid disease, any known abnormality of mineral metabolism (e.g., Wilson's disease, hemochromatosis), judged clinically to be at serious risk of suicide or violence, or currently taking any other medication with primarily central nervous system activity.

\subsection{Sample Recruitment}

Community based recruitment strategies are to be employed to recruit participants. Methods of recruitment include posters around Christchurch, at public health centers, and at the University of Canterbury, as well as advertising on social media outlets, namely Facebook.

Prospective participants will complete an initial screening questionnaire via the study website (http://bit.ly/nutritionandPMS). If the participant meets the preliminary inclusion criteria they are invited to attend further face-to-face screening at the university based laboratory.

\subsection{Treatment}

Participants will be randomized to receive either vitamin B6 or EMP+, the full list of ingredients and doses for both formulas can be viewed in Table 1. Numerous studies have used EMP+ for the treatment of mental health concerns, with no occurrences of significant adverse outcomes observed [30]. Vitamin B6, at very high doses, $2000 \mathrm{mg} /$ day has been shown to cause peripheral neuropathy (Joint Food Safety and Standards Group, 1997). However, the same, but reversible effects have been noted in participants taking $200 \mathrm{mg} /$ day [31]. Therefore, the current study is using $80 \mathrm{mg} /$ day, a dose that has proven effective on both physical and emotional symptoms of PMS without co-occurring side effects [17].

Table 1. Nutrient Information.

\begin{tabular}{|c|c|c|c|}
\hline \multicolumn{4}{|c|}{ EMPowerPlus Advanced Supplement Facts } \\
\hline \multicolumn{4}{|c|}{ Amount Per Serving (8 capsules) } \\
\hline Vitamin A (as retinyl palmitate) & 3072 IU & Calcium (as chelate) & $704 \mathrm{mg}$ \\
\hline Vitamin C (as ascorbic acid) & $320 \mathrm{mg}$ & Iron (as chelate) & $7.328 \mathrm{mg}$ \\
\hline Vitamin D (as cholecalciferol) & $768 \mathrm{IU}$ & Phosphorus (as chelate) & $448 \mathrm{mg}$ \\
\hline Vitamin E (as d-alpha tocopheryl succinate) & $192 \mathrm{IU}$ & Iodine (from Pacific kelp) & $108.8 \mathrm{mcg}$ \\
\hline Thiamin (as thiamin mononitrate) & $9.6 \mathrm{mg}$ & Magnesium (as chelate) & $320 \mathrm{mg}$ \\
\hline Riboflavin & $7.2 \mathrm{mg}$ & Zinc (as chelate) & $25.6 \mathrm{mg}$ \\
\hline Niacin (as niacinamide) & $48 \mathrm{mg}$ & Selenium (as chelate) & $108.8 \mathrm{mcg}$ \\
\hline Vitamin B6 (as pyridoxine hydrochloride) & $19.2 \mathrm{mg}$ & Copper (as chelate) & $3.84 \mathrm{mg}$ \\
\hline Folic acid & $768 \mathrm{mcg}$ & Manganese (as chelate) & $5.12 \mathrm{mg}$ \\
\hline Vitamin B12 (as methylcobalamin) & $480 \mathrm{mcg}$ & Chromium (as chelate) & $332.8 \mathrm{mcg}$ \\
\hline Biotin & $576 \mathrm{mcg}$ & Molybdenum (as chelate) & $76.8 \mathrm{mcg}$ \\
\hline Pantothenic acid (as calcium pantothenate) & $11.52 \mathrm{mg}$ & Potassium (as chelate) & $128 \mathrm{mg}$ \\
\hline \multicolumn{4}{|c|}{$\begin{array}{l}\text { Propriety blend: Choline bitartrate, DL-phenylalanine, citrus Bioflavonoids, Inositol, L-Glutamine, } \\
\text { L-Methionine, Grape seed extract, Gingko biloba leaf extract, germanium sesquioxide, Boron (as chelate), } \\
\text { Vanadium (as chelate), Nickel (as chelate). } \\
\text { Other ingredients: capsule shell (gelatin, titanium dioxide) microcrystalline cellulose, glycine, citric acid, } \\
\text { magnesium stearate, silicon dioxide, mineral wax }\end{array}$} \\
\hline
\end{tabular}


Table 1. Cont.

\begin{tabular}{cccc}
\hline \multicolumn{4}{c}{ Vitamin B6 Supplement Facts } \\
\hline Amount Per Serving (8 capsules) \\
\hline Vitamin B6 (as Pyridoxcine HCI) & $80 \mathrm{mg}$ & Riboflavin & $0.8 \mathrm{mg}$ \\
Acacia Gum & $2400 \mathrm{mg}$ & Cocoa Powder & $3167.2 \mathrm{mg}$ \\
\hline Other ingredients: maltodextrin, and steric acid & \\
\hline
\end{tabular}

\subsection{Study Procedure}

\subsubsection{Screening Assessment}

Prospective participants will complete a modified version of the Daily Record of Severity of Problems (DRSP) online to confirm the presence of menstrual related symptoms in the luteal phase of their menstrual cycle. They must meet the following to be included: (1) during the mid-follicular phase (day 6-10 after the onset of menses), their symptoms must be no more than "mild"; (2) in the week prior to menses, at least one emotional symptom i.e., depression, anxiety, affective liability, or anger must be "moderate" or higher; (3) during this phase they must also show a worsening in at least three out of the eleven symptoms covered in the DRSP questionnaire; (4) and finally these symptoms must have a "moderate" impact on their quality of life, as indicated by the participant's endorsement of one of three questions in the DRSP. These initial screening criteria are again to be confirmed during the double cycle baseline period. Judgments of "mild", "moderate" and so on will be based on the numerical value the participant ascribes to her symptom, where; $1=$ not at all, $2=$ minimal, $3=$ mild, $4=$ moderate, 5 = severe, $6=$ extreme.

Women who meet the above criteria will be invited to attend a meeting where current and past mood disorders are assessed using a psychiatric diagnostic interview (Structured Clinical Interview for DSM-5, Research Version) [32]. Any participants who are deemed to have a current mood disorder, other than Premenstrual Dysphoric Disorder (PMDD), will be excluded at this point. In instances where mood disorders are detected the clinical psychologist overseeing the study will be notified and referrals will be made to appropriate services, as deemed necessary by the psychologist. Likewise, any concerns arising during the baseline or treatment phase will be managed in the same manner; however, were a participant to develop a mood disorder during the treatment phase they would not be excluded from the study at this point. For an overview of the study process, please refer to Figure 1.

\subsubsection{Randomization and Allocation}

If an individual meets inclusion criteria, consents to participate in the study, and completes the two cycles of baseline, they will then be allocated the next available randomized participant number from a previously generated list. Participants are to be randomized in a 1:1 ratio to the micronutrients or vitamin B6 condition by a research assistant using block randomization [33]. The pharmacist will receive this randomization list. They will then package all capsules (micronutrient and vitamin B6) in plain white containers, labelled simply with the participant number and daily dose requirements. A sealed envelope containing the treatment allocation for each participant will be kept in a secure location, only to be opened in the case of an emergency (e.g., a serious deterioration in the participant's health), meaning the blind will be broken for that participant only. A separate randomization sheet is kept by the research assistant and is to be given to the statistician for analysis of the primary outcome at the end of data collection, and the researcher for analysis of the secondary outcomes. 


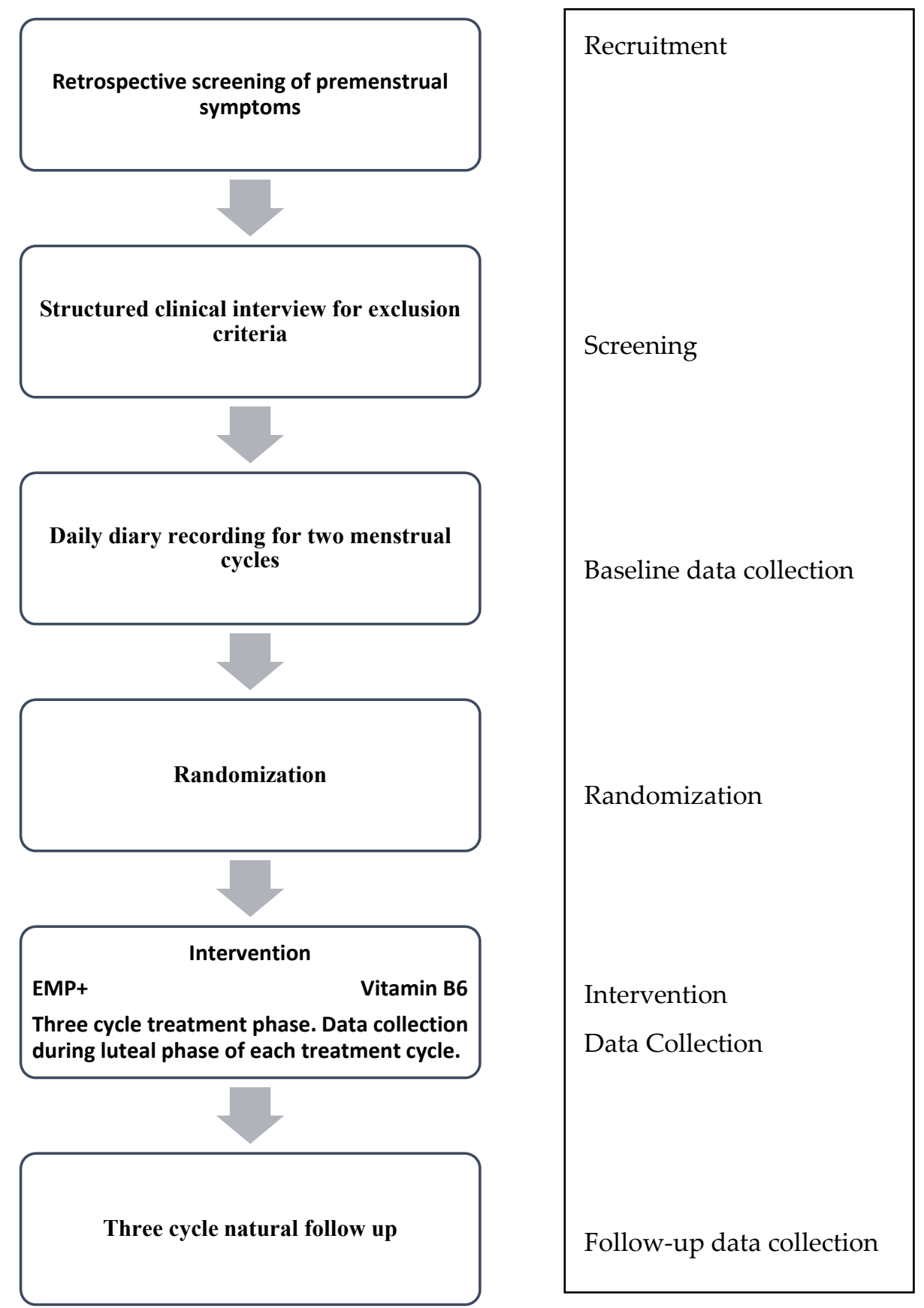

Figure 1. Study Process.

\subsubsection{Blinding}

Both participants and researchers will be blind to the treatment condition of each individual. Randomization will be conducted by a research assistant who will not be involved in any other aspect of the study. There is no difference between the appearance of the two supplements, and both contain riboflavin to ensure the change in urine color this vitamin causes is universal across the conditions. The dose of the vitamin B6 has been distributed across 8 pills such that the group randomized to vitamin B6 consumes the same number of pills. In order to fill the capsules, other inert ingredients are being given alongside vitamin B6 including; acacia gum, cocoa powder, maltodextrin, and steric acid, none of which are derived from wheat so the formula is safe for coeliacs to consume. Once all data are entered and analysis of the primary outcome measure is complete, the researchers will complete 
analysis of the secondary outcomes using a randomization list labelled A or B. Once all analyses are complete the blind will be broken and participants will be informed of their treatment allocation.

\subsubsection{Intervention}

Capsule dose will be titrated up over six days to the full dose of eight capsules/day (two doses of four capsules taken with water and food). Participants will continue to take the capsules for a full three menstrual cycles, as women with a cycle that lasts between 21-35 days are eligible for this study, the amount of time a participant is on active treatment will vary as a function of their cycle length. Based on previous clinical trials, participants usually describe a gradual effect of micronutrient supplements that reaches full effect within four weeks, therefore the three cycle treatment phase should, theoretically, afford enough time for treatment to reach its full effect.

During each of the three treatment cycles, participants will come into the University during the end of their PMS phase to complete questionnaires, receive new capsules and return old capsules and their DRSP diary. For each visit made to the University, following the initial baseline meeting, participants will receive a NZD 10 voucher able to be used at all major petrol stations across New Zealand. Treatment compliance is to be measured via counting and recording capsule consumption, whereby less than $80 \%$ consumption of the assigned dose will be considered non-compliance. Participants will return their remaining capsules at the end of the treatment phase in order for compliance to be assessed.

At the end of the treatment phase, participants will decide on their own course of treatment; taking vitamin B6, EMP+ or another active treatment of their choosing or discontinuing treatment altogether (they will remain blind to the treatment they were receiving). No treatment will be supplied by the study once participants have completed their three cycles of treatment, however, information on how to obtain vitamin B6 or EMP+ will be provided. Three cycles later participants will be contacted via email to complete a modified version of the DRSP online, as well as answer questions about their current PMS management, including treatment use over the preceding three cycles. The modified version of the DRSP will ask participants to rate their symptoms as usual on the 6 point scale; however, instead of completing the diary for each day of their PMS phase they will instead give an overall rating for each symptom at the end. Three cycles without treatment, if the participant chooses to do so, should ensure enough time to see a return of pre-treatment PMS scores should that happen.

\subsection{Data Collection and Outcome Measures}

Table 2 displays the outcome measures and time points at which they are to be collected. Demographic data including premenstrual syndrome history and previous and current mental health status is to be collected in the initial screening questionnaire. Self-report measures are obtained from each participant at baseline, and during each cycle of treatment to determine depressive, anxious, and stress symptoms, sleep quality, sexual satisfaction, and quality of life as well as general information on food and drug and alcohol intake.

\subsubsection{Primary Outcome Measure}

During the participant's first two menstrual cycles, daily data on premenstrual symptoms is to be collected via the Daily Record of Severity of Problems (DRSP) questionnaire [34]. The DRSP is a commonly used screening tool for premenstrual dysphoric disorder [2]. It provides a severity rating from 1 (not at all) to 6 (extreme) for 11 questions assessing premenstrual symptoms and asks a further three that look at the impact the symptoms have in terms of productivity, participation in normal activities, and relationship harmony [34]. For each cycle of treatment, participants will fill out the DRSP for the 10 days that correspond with their PMS phase. At natural follow up women will complete a modified version on the DRSP that will ask about their symptoms over the preceding week (again at the point in their cycle that corresponds with their PMS phase). 
Table 2. Schedule of measurements.

\begin{tabular}{|c|c|c|}
\hline Variable & Instrument & Time Point \\
\hline \multicolumn{3}{|l|}{ Self-report } \\
\hline Premenstrual symptoms & DRSP & $\begin{array}{l}\text { Two cycle baseline, each treatment } \\
\text { cycle, natural follow up }\end{array}$ \\
\hline $\begin{array}{l}\text { Psychiatric status } \\
\text { (mood disorders only) }\end{array}$ & $\begin{array}{l}\text { Structured Clinical Interview } \\
\text { (for DSM-5)-Research Version } \\
(\text { SCID-1/RV) }\end{array}$ & Screening \\
\hline Depression, anxiety, stress & $\begin{array}{l}\text { Depression and Anxiety Stress } \\
\text { Scales- } 42 \text { (DASS- } 42 \text { ) }\end{array}$ & Baseline, each treatment cycle \\
\hline Quality of life, wellbeing & $\begin{array}{l}\text { Women's Quality of Life } \\
\text { Questionnaire (WOMQOL) }\end{array}$ & Baseline, each treatment cycle \\
\hline Sleep quality & $\begin{array}{l}\text { Pittsburgh Sleep Quality } \\
\text { Index (PSQI) }\end{array}$ & Baseline, each treatment cycle \\
\hline Contextual stress & The Perceived Stress Scale (PSS) & Baseline, each treatment cycle \\
\hline Sexual satisfaction & $\begin{array}{l}\text { The New Sexual Satisfaction } \\
\text { Scale-Short From (NSSS-S) }\end{array}$ & Baseline, each treatment cycle \\
\hline $\begin{array}{l}\text { Diet quality, alcohol and } \\
\text { drug intake }\end{array}$ & $\begin{array}{l}\text { Drug, Alcohol, and Food } \\
\text { Questionnaire (DAF) }\end{array}$ & Baseline, each treatment cycle \\
\hline Treatment side effects & Side Effects Questionnaire & Baseline, each treatment cycle \\
\hline Treatment efficacy & $\begin{array}{l}\text { Clinical Global Impression scale } \\
\text { (CGI scale, Improvement scale) }\end{array}$ & End of treatment \\
\hline \multicolumn{3}{|l|}{ Observer report } \\
\hline $\begin{array}{l}\text { Change in } \\
\text { premenstrual symptoms }\end{array}$ & Modified DRSP & Baseline, end of treatment \\
\hline
\end{tabular}

*Clinician administered.

\subsubsection{Secondary Outcome Measures}

Third party symptom ratings: Participants, who agree, will be asked to provide details of a third-party observer who is able and willing to comment on the participant's PMS symptoms. Observers will do this via an online, modified version of the DRSP questionnaire that is to be filled out twice in the luteal phase; once at baseline and again at the end of the treatment.

Each cycle during the treatment phase and once at baseline, participants will fill out the following questionnaires; Depression and Anxiety Stress Scales-42 (DASS-42), Women's Quality of Life Questionnaire (WOMQOL), Pittsburgh Sleep Quality Index (PSQI), The Perceived Stress Scale (PSS), The New Sexual Satisfaction Scale-Short form (NSSS-S), a side effects questionnaire, and a drug, alcohol, and food questionnaire (DAF).

The DASS-42 is a 42 item self-report instrument designed to measure three related negative emotional states; depression, anxiety, and tension/stress [35]. It is used across a variety of clinical studies and as women with PMS/PMDD commonly experience symptoms measured by the DASS-42 it provides an additional useful tool for tracking psychometric change.

Contextual, common every day stress will be measured by the PSS 10-question version [36]. Participants are asked to rate the stressfulness of a number of events from the past week of their life in a modified version of the PSS. While there are three versions of the PSS, the current study will use the 10 -question version which has been well validated. Questions are rated on a $0-4$ point scale, focusing on relatively general stressors that pertain to every day events as opposed to large-scale incidents such as death or environmental disaster [36]. The PSS was found to be a good predictor of both health and health-related outcomes, and while it is correlated with depressive symptomatology it was found to 
measure an independent construct. In a sample of college students the PSS was found to have good test-retest reliability $(r=0.85)$, an important factor for this study.

The WOMQOL was specifically designed to assess a woman's quality of life in four domains: physical, psychological, social, and spiritual wellbeing [37]. Forty questions have three possible answers; Yes, No, or Not Applicable with higher overall scores indicating a better quality of life. Quality of Life scales are frequently used in chronic illness and disease studies as a measure of disease impact and treatment response [3].

The New Sexual Satisfaction Scale-Short Form (NSSS-S) is comprised of 12 items which are measured on a 5-point scale [38]. Two factors are covered in this questionnaire; personal experiences and sensations, and partner's general behaviors and sexual activity, with higher scores indicating greater sexual satisfaction [38]. The form shall be modified slightly in time period; instead of asking about sexual satisfaction over the last six months, participants will assess their satisfaction over the last week. Only those women who are currently sexually active (excluding solo sexual activity) will be asked to fill out the questionnaire.

Pittsburgh Sleep Quality Index (PSQI) measures sleep quality and disturbances over a one month period [39]. There are 19 questions which yield seven different measures; subjective sleep quality, sleep latency, sleep duration, habitual sleep efficiency, sleep disturbances, use of sleeping medication, and daytime dysfunction. These scores can then sum together to give an overall sleep quality score. The PSQI has been found to have sound reliability and validity [39].

Drug, Alcohol, and Food Questionnaire (DAF). Questions regarding dietary patterns over the previous week will be assessed using a questionnaire modified from Baker, et al. [40]. In this study, a healthy eater will be defined as someone who eats in a balanced way, eats three meals a day, doesn't eat too much junk food, eats moderate amounts, and stops eating when full. Participants are also asked to indicate from 1 (<once a week) to 5 (daily) how often over the previous 2 weeks they ate breakfast, ate a balanced meal, ate even when full, ate lots of fruits and vegetables, and ate fast foods or snack foods such as potato chips or candy bars. Three items are reverse scored. They will also be asked about average daily servings of fruit and vegetables (from 1 (<one serving) to 5 ( 4 or more servings)), and to indicate from 1 (not very healthy) to 7 (very healthy) how healthy they think their diet is. Total scores will range from 9 to 47 , with a higher score indicative of a healthier diet. These specific questions were recently adapted and validated by Kuijer and Boyce [41]. In the validation study, Kuijer and Boyce showed that the questions were correlated with a 2 week diary report of those behaviors. Moreover, the retrospective recall was found to be a fairly accurate estimate of the eating behaviors as reported during the diary period. The summing of the items has been used successfully in other studies such that a higher score on the summed scale indicates healthier eating behaviors (Cronbach's alpha 0.67) [41]. In the course of answering questions on food intake, participants will also be asked about alcohol and drug consumption over the preceding week.

A scale assessing common side effects for both treatment groups will be administered throughout the study, including baseline. Participants will be asked to indicate whether any symptoms commonly identified as side effects were experienced during treatment and then asked what actions they have taken to remedy them.

In the final set of questionnaires, at the end of the third cycle of intervention, participants will be asked to complete the Clinical Global Impression scale (CGI scale) [42]. The CGI scale is used in numerous clinical studies to evaluate the global efficacy and safety of a medication [43]. Participants will judge their own improvement at the end of the trial on the Clinical Global Impression-Improvement scale (CGI-I) in a self-rated version rather than a clinician rated scale.

\subsection{Data Management}

All study data is to be contained in locked storage systems; either a password protected computer system at the University of Canterbury or on a web-based data collection system (www.canterbury. 
qualtrics.com) for electronic documents, while hard copies will be kept in secure filing cabinets at the university.

\subsection{Study Integrity}

The trial prospectively registered under the Australian and New Zealand Clinical Trials Registry $($ ANZCTR). Trial Identification $=$ ACTRN12615000131550.

Universal Trial Number $($ UTN $)=$ U1111-1164-2407.

Ethics approval was granted through the University of Canterbury Human Ethics Committee: HEC 2014/129, on 13 November 2014. Approval was updated on 4 February 2015 following trial amendments. Written informed consent is to be granted by all participants before entry into this study.

\subsection{Sample Size}

Determining the sample size was a challenge based on the diversity of studies that have thus far been conducted. We are aiming to recruit 40 participants per group, making a total of 80 participants. The final sample size was calculated via a sample size calculation where the best estimate of the effect size (ES) of vitamin B6 on psychiatric symptoms was 0.36 [17], while EMP+ produced (in a conservative estimate) an ES of 0.64 on mood symptoms in a blinded trial [44]. Yet in an open label trial the reported effect size was 1.96 [45]. To detect a medium ES of 0.6 in this study, a total of 72 participants are needed. This number has been increased to 80,40 per-group, to allow for attrition. We have chosen to detect a medium effect as we believe this will be clinically relevant for those women choosing to use natural supplements in treating PMS. Based on the available data is it reasonable to expect to see a medium ES difference between the two treatments; currently estimates for mood in blinded micronutrient trials appears to be medium to large [44].

\subsection{Data Analyses}

The first participant enrolled in this study on the 1 March 2014, final data collection is expected to be completed by May 2017.

Descriptive statistics including age, cycle length, menstruation length, duration of premenstrual complaints and occupation, will be documented in order to compare the two treatment group's homogeneity to each other and to the wider population. Two-tailed $t$-tests or ANOVA will be used to identify any group differences.

The primary efficacy analysis will assess average treatment group differences, as well as individual differences for the primary outcome measure (DRSP) using a repeated measures design. Repeated-measures ANCOVA will be used to assess changes from baseline to end of treatment between both treatment groups, using the baseline level as the covariate. Time series graphs will also be constructed to show the intensity and trajectory of change in symptom patterns overtime at both an individual and treatment group level. Finally, modified Brinley Plots [46] will be constructed to show overall change, along with Reliable Change for each individual, overall \%Reliable Positive Change, and Cohen's d Effect Size. Pre and post treatment data points for each participant will be placed onto a scatter plot, where the direction of desired change and clinical cut-offs are indicated to assist interpretation. Separate plots will be drawn for each secondary measure of sleep, stress, sexual satisfaction, quality of life, and symptoms of depression, anxiety and stress.

Intention to treat (ITT) analysis will be employed, thus all participants will be included regardless of whether they complied with capsule consumption. Treatment adherence will be based on total treatment consumption; less than $80 \%$ will be considered non-treatment adherence. Per-protocol analysis will be performed as secondary analysis of DRSP change, whereby participants with protocol violations (drop-out, incomplete treatment consumption) will be excluded. Those participants who drop out will be classified based on treatment group and reason for discontinuation. Categorical outcome of drop out will then be compared between the two treatment groups using Chi-square tests with a 95\% confidence interval and Odds Ratios. 
Hierarchical linear regression will be used to investigate baseline stress sensitivity as a possible mediator/moderator of treatment outcome. Effect sizes will be calculated using Cohen's d guidelines, while an alpha level of 0.05 will be set on all tests of treatment effects.

\section{Discussion}

Many women seek a natural solution to treating their monthly premenstrual symptoms. Single ingredient treatments have shown some promise in improving both emotional and physical symptoms of PMS [11], yet there is a gap in our knowledge about the efficacy of combined micronutrients. Previous research in this area has focused on multi-ingredient formulas high in specific vitamins, clouding the effects of a broad spectrum treatment [27]. The current study aims to assess the efficacy of a broad spectrum micronutrient formula, and compare it to an already established natural treatment; answering in the process the question of "is a multi-ingredient formula better than a single vitamin treatment for PMS?" Additionally, a secondary aim is to assess and compare the wide ranging effect of both treatments on sleep, sexual satisfaction, quality of life, stress, anxiety, and depression. Stress sensitivity, conceptualized as an individual's baseline stress level and their response to stress, is to be further investigated for its possible moderating/mediating effect on PMS symptoms.

Acknowledgments: This study is being funded through the University of Canterbury's Department of Psychology internal PhD grants.

Author Contributions: All three authors conceived and designed this experiment. Data is to be collected by Hannah Retallick-Brown. The majority of the analysis is to done by Hannah Retallick-Brown with contributions from both Julia Rucklidge and Neville Blampied including the use of detailed analysis tools designed by Neville Blampied. Hannah Retallick-Brown wrote this paper.

Conflicts of Interest: The authors declare no conflict of interest

\section{References}

1. Chavez-MacGregor, M.; van Gils, C.H.; van der Schouw, Y.T.; Monninkhof, E.; van Noord, P.A.; Peeters, P.H. Lifetime cumulative number of menstrual cycles and serum sex hormone levels in postmenopausal women. Breast Cancer Res. Treat. 2008, 108, 101-112. [CrossRef] [PubMed]

2. Lustyk, M.K.B.; Gerrish, W.G. Premenstrual Syndrome and Premenstrual Disorder: Issues of Quality of Life, Stress and Exercise; Springer: London, UK, 2010; Volume 115, pp. 1952-1975.

3. Rapkin, A.J.; Winer, S.A. Premenstrual syndrome and premenstrual dysphoric disorder: Quality of life and burden of illness. Expert Rev. Pharmacoecon. Outcomes Res. 2009, 9, 157-170. [CrossRef] [PubMed]

4. American Psychiatric Association. Diagnostic and Statisticl Manual of Mental Disorders, 5th ed.; American Psychiatric Publishing: Arlington, VA, USA, 2013.

5. American College of Obstetricians and Gynecologists. ACOG Practice Bulletin: Premenstrual Syndrome. National Guideline; Clearninghouse: Washington, DC, USA, 2000.

6. Marjoribanks, J.; Brown, J.; O'Brien, P.M.; Wyatt, K. Selective serotonin reuptake inhibitors for premenstrual syndrome. Cochrane Database Syst. Rev. 2013, 7, CD001396.

7. Brown, J.; Marjoribanks, J.; O’Brien, P.M.; Wyatt, K. Selective serotonin reuptake inhibitors for premenstrual syndrome. Cochrane Database Syst. Rev. 2009, 15, CD001396.

8. Dimmock, P.W.; Wyatt, K.M.; Jones, P.W.; Shaughn O'Brien, P.M. Efficacy of selective serotonin-reuptake inhibitors in premenstrual syndrome: A systematic review. Lancet 2000, 356, 1131-1136. [CrossRef]

9. Bhatia, S.C.; Bhatia, S.K. Diagnosis and Treatment of Premenstrual Dysphoric Disorder. Am. Family Physician 2002, 66, 1239-1249.

10. Jarvis, C.I.; Lynch, A.M.; Morin, A.K. Management Strategies for Premenstrual Syndrome/Premenstrual Dysphoric Disorder. Ann. Pharmacother. 2008, 42, 965-978. [CrossRef] [PubMed]

11. Cunningham, J.; Yonkers, K.A.; O’Brien, S.; Erikson, E. Update on research and treatment of premenstrual dysphoric disorder. Harv. Rev. Psychiatry 2009, 17, 120-137. [CrossRef] [PubMed]

12. American College of Obstetricians and Gynaecologists Premenstrual Syndrome: Commonly Asked Questions. Available online: http://www.acog.org/ / media/For\%20Patients $/$ faq057.pdf?dmc $=1 \& t s=$ 20140703T0000427282 (accessed on 3 March 2016). 
13. Kaplan, B.J.; Crawford, S.G.; Field, C.J.; Simpson, J.A. Vitamins, minerals, and mood. Psychol. Bull. 2007, 133, 747-760. [CrossRef] [PubMed]

14. Bussell, G. Pre-menstrual syndrome and diet. J. Nutr. Environ. Med. 1998, 8, 65-75.

15. Bendich, A. The Potential for Dietary Supplements to Reduce Premenstrual Syndrome (PMS) Symptoms. J. Am. Coll. Nutr. 2000, 19, 3-12. [CrossRef] [PubMed]

16. Doll, H.; Brown, S.; Thurston, A.; Vessey, M. Pyridoxine (vitamin B6) and the premenstrual syndrome: A randomized crossover trial. J. R. Coll. Gen. Pract. 1989, 39, 364-368. [PubMed]

17. Kashanian, M.; Mazinani, R.; Jalalmanesh, R. Pyridoxine (vitamin B6) therapy for premenstrual syndrome. Int. J. Gynecol. Obs. 2007, 96, 43-44. [CrossRef] [PubMed]

18. Wyatt, K.M.; Dimmock, P.W.; Jones, P.W.; Shaughn O'Brien, P.M. Efficacy of vitamin B-6 in the treatment of premenstrual syndrome: Systematic review. Br. Med. J. 1999, 318, 1375-1381. [CrossRef]

19. Chocano-Bedonya, P.O.; Manson, J.E.; Hankinson, S.E.; Willet, W.C.; Johnson, S.R.; Chasan-Taber, L.; Ronnenberg, A.G.; Bigelow, C.; Bertone-Johnson, E.R. Dietary B vitamin intake and incident premenstrual syndrome. Am. J. Clin. Nutr. 2011, 93, 1080-1086. [CrossRef] [PubMed]

20. Mertz, W. A balanced approach to nutrition for health: The need for biologically essential minerals and vitamins. J. Am. Diet. Assoc. 1994, 94, 1259-1262. [CrossRef]

21. Thys-Jacobs, S.; Starkey, P.; Bernstein, D.; Tian, J. Calcium carbonate and the premenstrual syndrome: Effects on premenstrual and menstrual symptoms. Premenstrual Syndrome Study Group. Am. J. Obs. Gynecol. 1998, 179, 444-452. [CrossRef]

22. London, R.; Murphy, L.; Kitlowski, K.; Reynolds, M. Efficacy of alpha-tocopherol in the treatment of the premenstrual syndrome. J. Reprod. Med. 1987, 32, 400-404. [PubMed]

23. Walker, A.R.; de Souza, M.C.; Vickers, M.F.; Abeyasekera, S.; Collins, M.L.; Trinca, L.A. Magnesium supplementation alleviates premenstrual symptoms of fluid retention. J. Womens Health 1998, 7, 1157-1165. [CrossRef] [PubMed]

24. De Souza, M.C.; Walker, A.F.; Robinson, P.A.; Bolland, K. A synergistic effect of a daily supplement for 1 month of $200 \mathrm{mg}$ magnesium plus $50 \mathrm{mg}$ vitamin B6 for the relief of anxiety-related premenstrual symptoms: A randomized, double-blind, cross-over study. J. Womens Health Gender-Based Med. 2000, 9, 131-139. [CrossRef] [PubMed]

25. Kaplan, B.J.; Rucklidge, J.J.; Romijn, A.; McLeod, K. The emerging field of nutritional mental health: Inflammation, the microbiome, oxidative stress, and mitochondrial function. Clin. Psychol. Sci. 2015, 3, 1-17. [CrossRef]

26. Rucklidge, J.J.; Kaplan, B.J. Broad-spectrum micronutrient formulas for the treatment of psychiatric symptoms: A systematic review. Expert Rev. Neurother. 2013, 13, 49-73. [CrossRef] [PubMed]

27. Chakmakjian, Z.H.; Higgins, C.E.; Abraham, G.E. The effect of a nutritional supplement, OPTIVITE ${ }^{\circledR}$ for women, on premenstrual tension syndromes: II. Effect on symptomatology, using a double blind cross-over design. J. Appl. Nutr. 1985, 37, 12-17.

28. Stewart, A.C.; Tooley, S.H.; Tooke, J.; Stewart, M.; Paterson-Jones, S. Effect of nutritional programme on premenstrual syndrome and work efficiency. Complement. Ther. Med. 1993, 1, 68-72. [CrossRef]

29. London, R.S.; Bradley, L.; Chiamori, N.Y. Effect of a nutritional supplement on premenstrual symptomatology in women with premenstrual syndrome: A double-blind longitudinal study. J. Am. Coll. Nutr. 1991, 10, 494-499. [CrossRef] [PubMed]

30. Simpson, J.S.A.; Crawford, S.G.; Goldstein, E.T.; Field, C.; Burgess, E.; Kaplan, B.J. Systematic review of safety and tolerability of a complex micronutrient formula used in mental health. BMC Psychiatry 2011, 11, 62. [CrossRef] [PubMed]

31. Malmgren, R.; Collins, A.; Nilsson, C.G. Platelet serotonin uptake and effects of vitamin B6-treatment in premenstrual tension. Neuropsychobiology 1987, 18, 83-86. [CrossRef] [PubMed]

32. First, M.B.; Williams, J.B.W.; Karg, R.S.; Spitzer, R.L. Structured Clinical Interview for DSM-5-Research Version (SCID-5 for DSM-5, Research Version; SCID-5-RV); American Psychiatric Association: Arlington, VA, USA, 2015.

33. Dallal, G.E. Randomization. Available online: http:/ / www.randomization.com (accessed on 30 March 2013).

34. Endicott, J.; Nee, J.; Harrison, W. Daily Record of Severity of Problems (DRSP): Reliability and validity. Arch. Women Health 2006, 9, 41-49. [CrossRef] [PubMed] 
35. Lovibond, P.F.; Lovibond, S.H. The structure of negative emotional states: Comparison of the Depression Anxiety Stress Scales (DASS) with the Beck Depression and Anxiety Inventories. Behav. Res. Ther. 1995, 33, 335-343. [CrossRef]

36. Cohen, S.; Kamarck, T.; Mermelstein, R. A global measure of perceived stress. J. Health Soc. Behav. 1983, 24, 385-396. [CrossRef] [PubMed]

37. Gehlert, S.; Chang, C.-H.; Bock, R.D.; Hartlage, A.A. The WOMQOL instrument measured quality of life in women of reproductive age with no known pathology. J. Clin. Epidemiol. 2006, 59, 525-533. [CrossRef] [PubMed]

38. Stulhofer, A.; Busko, V.; Brouillard, P. The new sexual satisfaction scale and its short form. In Handbook of Sexuality-Related Measures; Fisher, T.D., Davis, C.M., Yarber, W.L., Davis, S.L., Eds.; Sage: Thousand Oaks, CA, USA, 2011; pp. 530-532.

39. Buysse, D.J.; Reynolds, C.F.; Monk, T.H.; Berman, S.R.; Kupfer, D.J. The Pittsburgh sleep quality index: A new instrument for psychiatric practice and research. Psychiatry Res. 1988, 28, 193-213. [CrossRef]

40. Baker, C.W.; Little, T.D.; Brownell, K.D. Predicting adolescents eating and activity behaviors. The role of social norms and personal agency. Health Psychol. 2003, 22, 189-198. [CrossRef] [PubMed]

41. Kuijer, R.G.; Boyce, J.A. Chocolate cake. Guilt or celebration? Associations with healthy eating attitudes, perceived behavioural control, intentions and weight-loss. Appetite 2014, 74, 48-54. [CrossRef] [PubMed]

42. National Institute of Mental Health. Clinical global impressions (028 CGI). In ECDEU Assessment Manual for Psychopharmacology; Guy, W., Ed.; National Institute of Mental Health: Rockville, MD, USA, 1976; pp. $218-222$.

43. Lauritzen, C.; Reuter, H.D.; Repges, R.; Böhnert, K.-J.; Schmidt, U. Treatment of premenstrual tension syndrome with vitex agnus castus controlled, double-blind study versus pyridoxine. Phytomedicine 1997, 4, 183-189. [CrossRef]

44. Rucklidge, J.J.; Frampton, C.M.; Gorman, B.; Boggis, A. Vitmain-mineral treatment of attention-deficit hyperactivity disorder in adults: Double-blind randomised palcebo-controlled trial. Br. J. Psychiatry 2014, 204, 306-315. [CrossRef] [PubMed]

45. Rucklidge, J.; Taylor, M.; Whitehead, K. Effect of micronutrients on behavior and mood in adults With ADHD: Evidence from an 8-week open label trial with natural extension. J. Atten. Disord. 2011, 15, 79-91. [CrossRef] [PubMed]

46. Blampied, N.M. Analyzing therapeutic change using modified Brinley plots: History, construction, and interpretation. Behav. Ther. 2016, in press. [CrossRef] 